\title{
Field camera unit of the WSO-UV mission
}

Sachkov, M., Sichevsky, S., Shustov, B., Shugarov, A., Shmagin, V., et al.

M. Sachkov, S. Sichevsky, B. Shustov, A. Shugarov, V. Shmagin, S. losipenko, R. Arkhangelsky, E. Kanev, A. I. Gomez de Castro, J. C. Vallejo, "Field camera unit of the WSO-UV mission," Proc. SPIE 11444, Space Telescopes and Instrumentation 2020: Ultraviolet to Gamma Ray, 1144474 (13 December 2020); doi: 10.1117/12.2562962

SPIE. Event: SPIE Astronomical Telescopes + Instrumentation, 2020, Online Only 


\title{
Field Camera Unit of the WSO-UV mission
}

\author{
M. Sachkov ${ }^{* a}$, Sichevsky $S^{\text {a }}$., Shustov $B^{\text {a }}$., Shugarov ${ }^{\text {a }}{ }^{\text {., Shmagin }} V^{\text {a }}$., Iosipenko $S^{a}$., \\ Arkhangelsky R ${ }^{\mathrm{a}}$., Kanev $\mathrm{E}^{\mathrm{a}}$., A.I. Gómez de Castro ${ }^{\mathrm{b}}$, Vallejo J.C ${ }^{\mathrm{b}}$ \\ ${ }^{a}$ Institute of Astronomy of Russian Academy of Sciences, Pyatnitskaya str., 48, Moscow, 119017, \\ Russia; \\ ${ }^{\mathrm{b}}$ AEGORA Research Group, Fac. CC. Matematicas, Universidad Complutense, Plaza de Ciencias 3, \\ 28040, Madrid, Spain
}

\begin{abstract}
The main instrument of the space mission "Spectr-UF" (World Space Observatory-Ultraviolet) is a large space telescope to work in the UV wavelength domain $(115-310 \mathrm{~nm})$. The WSO-UV telescope feeds in its focal plane two main instruments: unit of spectrographs WUVS, and field camera unit FCU, as well as Fine Guidance System (FGS). The imaging instrument FCU onboard WSO-UV will be the first UV camera to be flown to a geosynchronous orbit. Here we provide a brief description on updated FCU instrument and its current status.
\end{abstract}

Keywords: ultraviolet spectral range, imaging, space telescope, WSO-UV

\section{INTRODUCTION}

After the launch in 2019 the second astrophysical observatory "Spektr-RG" of the "Spektr" series of the Russian Federal Space Program (the first was the "Spektr-Radioastron" mission, 2011 -- 2019), the "Spektr-UF" mission project (World Space Observatory - Ultraviolet, WSO-UV) became the next to be launched. The WSO-UV observatory includes a 170 $\mathrm{cm}$ aperture telescope capable of high-resolution spectroscopy and long slit low-resolution spectroscopy with the WUVS (WSO-UV Spectrographs) instrument. Moreover UV imaging will be available with the Field Camera Unit (FCU) instrument. The general information on the mission as well as updated information on the WSO-UV systems/subsystems can be found in the SPIE proceedings ${ }^{1,2,3}$. The key scientific drivers of the WSO-UV project are described in papers ${ }^{4,5,6}$ and in some others. Observation time of the observatory will be divided between three areas: the Core program (approximately 50\% of the total time during the first two years on orbit), National programs of participating countries (approximately $40 \%$ of the total observation time, in proportion to the financial contributions of countries to the project the first two years on orbit) and the Open program (open Program, implementation of applications of high scientific significance, including from scientists from countries not participating in the project). First of all, the multi-purpose observatory WSO-UV will be used to realize approved applications of the Core Program, the scientific justification of which, and the corresponding links are given in detail in the paper by Boyarchuk et $\mathrm{al}^{7}$. The science management plan of WSO-UV was presented in a paper by Malkov et $\mathrm{al}^{8}$. In the following sections we present a current status of the FCU instrument as well as major charges in its design.

\section{FCU SCIENCE CASE}

The Core Program of WSO-UV will be concentrated on the following tasks: Universe evolution studies, Studies of accretion processes, Star formation studies, Studies of (exo)planetary atmospheres.

*msachkov@inasan.ru; phone +7-495-951-1279

Space Telescopes and Instrumentation 2020: Ultraviolet to Gamma Ray, edited by Jan-Willem A. den Herder

Shouleh Nikzad, Kazuhiro Nakazawa, Proc. of SPIE Vol. 11444, 1144474 · (C) 2020 SPIE

CCC code: $0277-786 X / 20 / \$ 21 \cdot$ doi: $10.1117 / 12.2562962$ 
As it was described in our previous paper ${ }^{9}$, the main scientific issues of the FCU are (but not limited by):

(1) Study of planetary nebulae;

(2) SuperNovae behavior in the UV;

(3) Study of short timescale (order of $40 \mathrm{~ms}$ ) variable sources;

(4) Asteroseismology;

(5) Exoplanetary atmospheres;

(6) Protostellar Jets;

(7) The Galactic globular clusters and variables.

WSO-UV will be one of the major workhorse for exo- planet observations. The current exoplanet research can be divided into five major aspects: (i) planet detection, (ii) planet formation and migration, (iii) planets' physical properties, (iv) planets' chemical properties (including biomarkers), and (v) planets as a tool to study the properties of the host stars. The WSO-UV mission will be a crucial tool for the study of the last four aspects.

\section{FCU CURRENT DESIGN}

The main task of the FCU instrument is obtaining high-resolution images and low-resolution spectra in the Far UV (115$180 \mathrm{~nm})$ and Near UV (180 -300 nm) wavelength range. The number of optical elements and mobile elements in FCU should be minimal to increase its reliability.

Functionally, the FCU consists of two channels: NUV (near ultraviolet channel) and FUV (vacuum ultraviolet channel):

(1) FUV channel operating range of wavelengths shouldn't be narrower than 115 to $176 \mathrm{~nm}$;

The FUV channel is equipped with a MCP (micro channel plates) detector, which is supplied by Spain. Main features of this channel are:

a) diffractive image quality;

b) highly sensitive photon counting mode;

c) high time resolution.

(2) NUV channel operating range of wavelengths shouldn't be narrower 174 to $305 \mathrm{~nm}$.

The NUV channel is equipped with a CCD manufactured by Teledyne-e2V, sensitive to the range from 174 to $305 \mathrm{~nm}$ (with a possible extension to $1000 \mathrm{~nm}$ ). Main features of this channel are:

a) large field of view;

b) large dynamic range.

Main characteristics of the FCU and similar cameras of the Hubble Space Telescope are shown in the Table 1.

Comparison of FCU and HST cameras allows us to highlight the main advantages of FCU. Compared to HST cameras, the FCU will provide a lower, though still very high, angular resolution. However, a large-format CCD together with a single - mirror scheme will allow to achieve the larger field of view, about 6 arc sec in the NUV channel. This will provide a very high efficiency of the FCU in terms of "discover efficiency", significantly exceeding similar HST cameras in this parameter. It should be noted that the FCU will be the first scientific UV instrument (camera) for highresolution observation in geosynchronous orbit, i.e. above the main mass of the geocorona.

In general, it can be noted that the FCU has a high sensitivity, a large field of view, and allows to work not only in the $\mathrm{UV}$, but also potentially in the optical and near-IR ranges. 
Table 1. Comparison of the characteristics of FCU (FUV and NUV channels) and HST cameras SBC (HST/ACS/SBC) и UVIS (HST/WFC3/UVIS).

\begin{tabular}{|l|l|l|l|l|}
\hline Characteristic & FUV FCU & NUV FCU & SBC & UVIS \\
\hline Detector type & MCP & CCD & MCP, MAMA & CCD \\
\hline Wavelength range, $\mathbf{n m}$ & $115-190$ & $170-944$ & $115-170$ & $200-1000$ \\
\hline Effective square $\mathbf{m}^{\mathbf{2}}$ & 0,068 & 0,270 & 0,180 & 0,450 \\
\hline Field of vie, " & $121 \times 121$ & $597 \times 451$ & $34,59 \times 30,8$ & $162 \times 162$ \\
\hline Scale, "/pixel & 0,047 & 0,146 & $0,033 \times 0,030$ & 0,0395 \\
\hline Detector size, mm & 30 & $49 \times 37$ & 25 & $61 \times 61$ \\
\hline Detector size, pxl & $2 \mathrm{k} \times 2 \mathrm{k}$ & $4 \mathrm{k} \times 3 \mathrm{k}$ & $1 \mathrm{k} \times 1 \mathrm{k}$ & $4 \mathrm{k} \times 4 \mathrm{k}$ \\
\hline Number of filters & $<10$ & $<15$ & 6 & 62 \\
\hline Number of prisms & 2 & - & 2 & - \\
\hline
\end{tabular}

The Conceptual design of the Field Camera Unit instrument is presented on the Fig 1.

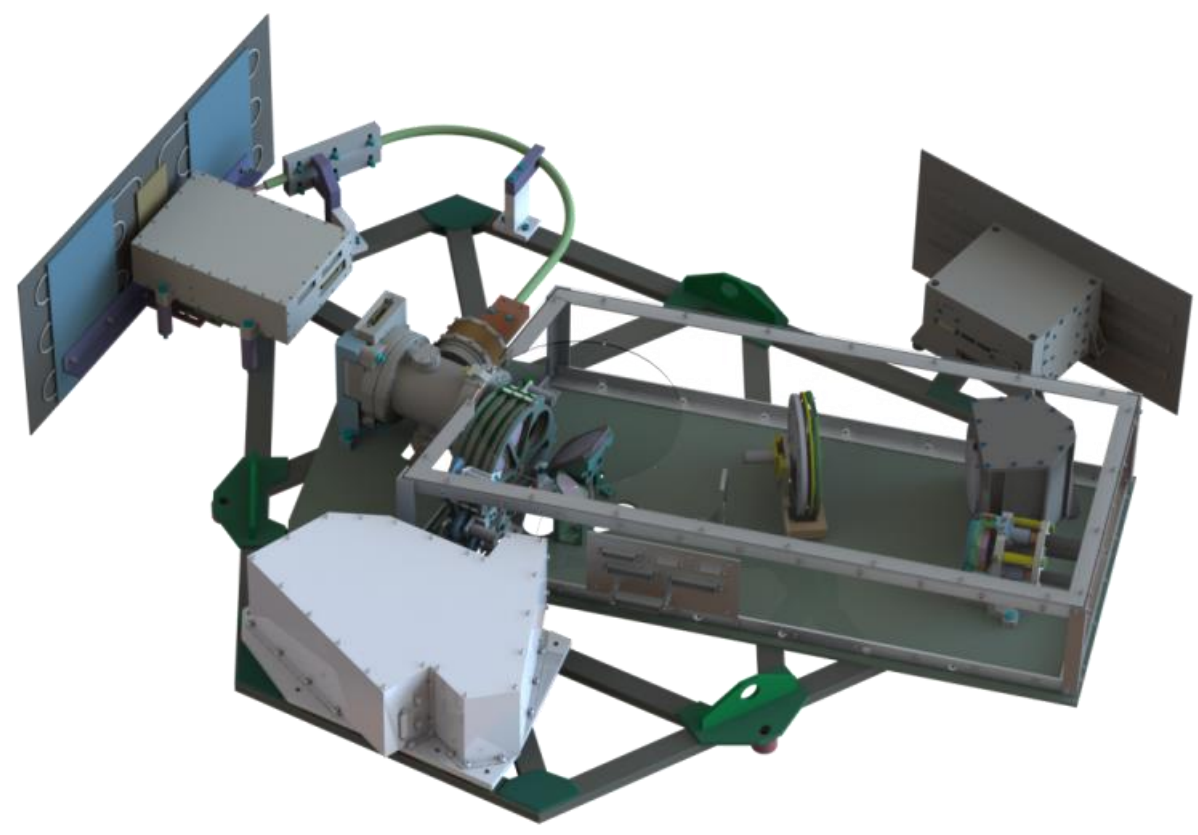

Figure 1. General view of FCU.

\section{NEAR UV CHANNEL}

Field of view of NUV channel is situated in the center of the field of view of the telescope T-170M. There is only one reflection in this channel: the flat mirror located on the telescope optical axis at an angle of $45^{\circ}$ (Fig 2$)$. To select the spectral range, a filter in the form of a plane-parallel plate is used, located between the plane mirror and the photodetector. The CCD detector is identical to that is used in WSO-UV spectrographs. 


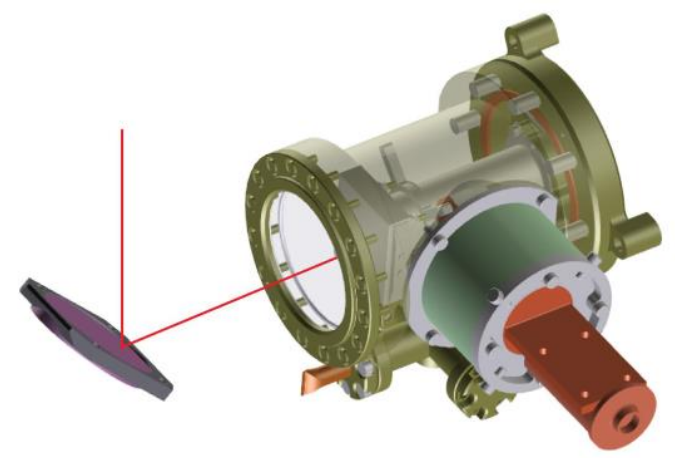

Figure 2. Schematic diagram of the optical ray path (red line) in the NUV channel.

The choice of filters is of great importance for the scientific effectiveness of the channel. There are three filter wheels in the NUV channel. Each wheel has 6 slots, 5 will be used for placing filters and one is empty. Thus, the total number of filters placed in the UV channel is 15. The very preliminary list of filters is presented in the Table 2.

Table 2. Preliminary filter list on NUV channel. $\lambda$ min is the minimal wavelength, $\lambda$ max is the maximum wavelength, CWL is the central wavelength and FWHM is the full width at the half-amplitude level

\begin{tabular}{|l|l|l|l|l|l|}
\hline \multicolumn{1}{|c|}{ Filter } & \multicolumn{1}{c|}{ Filter type } & $\lambda$ min, $\mathrm{nm}$ & \multicolumn{1}{c|}{$\lambda$ max, $\mathrm{nm}$} & \multicolumn{1}{c|}{ CWL, $\mathrm{nm}$} & \multicolumn{1}{c|}{ FWHM, $\mathrm{nm}$} \\
\hline F255W & Wide-band filter & 230 & 280 & 255.0 & 50.0 \\
\hline F336W & Wide-band filter & 311 & 361 & 336.0 & 50.0 \\
\hline F555W & Wide-band filter & 480 & 630 & 555.0 & 150.0 \\
\hline F438W & Wide-band filter & 403 & 473 & 438.0 & 70.0 \\
\hline F606W & Wide-band filter & 491 & 721 & 606.0 & 230.0 \\
\hline F814W & Wide-band filter & 684 & 944 & 814.0 & 260.0 \\
\hline F185W & Wide-band filter & 170 & 200 & 185.0 & 30.0 \\
\hline F232N & Narrow-band filter & 229,8 & 234,8 & 232.3 & 5.0 \\
\hline F280N & Narrow-band filter & 277,5 & 282,5 & 280.0 & 5.0 \\
\hline F308N & Narrow-band filter & 305,5 & 310,5 & 308.0 & 5.0 \\
\hline F656N & Narrow-band filter & 653,8 & 658,8 & 656.3 & 5.0 \\
\hline F673N & Narrow-band filter & 668 & 678 & 673.0 & 10.0 \\
\hline F250LP & Longpass filter & 250 & - & & \\
\hline Gbp & Gaia BP filter & 328 & 671 & & \\
\hline Glnuv & GALEX NUV filter & 169 & 300 & & \\
\hline
\end{tabular}

\section{FAR UV CHANNEL}

The schematic diagram of the path of optical rays in the Far UV channel is shown in the Figure 3. 


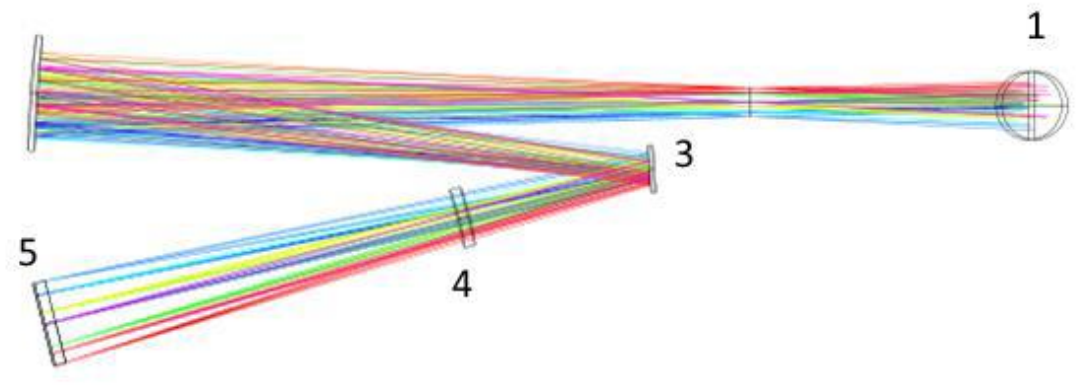

Figure 3. Optical scheme of the FUV channel. 1) Flat mirror; 2) Spherical mirror; 3) Aspherical mirror; 4) Spectral filter; 5) Photodetector.

The VUVV channel includes 8 filter slots and contains a set of 5 filters, 2 prisms and one free slot. Only long pass filters will be used in this channel. These filters allow to pass only a part of the spectrum with wavelengths greater than the cutoff wavelength (the long-wave part of the spectrum). Using a combination of two such filters allows to "cut" the spectral bands of the desired width to solve the current scientific problem.

Implementation of the mode of slit-less spectroscopy in the FUV channel is possible with the use of prisms, which are wedge-shaped meniscus lenses installed in the filter wheel. The use of prisms, in contrast to diffraction gratings, allows to register not a single section of the spectrum, but the entire spectrum at once.

Preliminary list of filters and prisms is presented in the Table3.

Table 3. Preliminary list of filters and prisms of FUV channel. $\lambda$ min is the minimal wavelength.

\begin{tabular}{|l|l|l|l|}
\hline Filter/Prism & Filter type & $\lambda$ min, $\mathrm{nm}$ & Material \\
\hline F115LP & Longpass filter & 115 & $\mathrm{MgF} 2$ \\
\hline F125LP & Longpass filter & 125 & $\mathrm{CaF} 2$ \\
\hline F140LP & Longpass filter & 140 & $\mathrm{BaF} 2$ \\
\hline F150LP & Longpass filter & 150 & $\mathrm{SiO} 2$ \\
\hline F165LP & Longpass filter & 165 & Fused silica \\
\hline PR115 & Prism & 115 & $\mathrm{MgF} 2$ \\
\hline PR140 & Prism & 140 & $\mathrm{BaF} 2$ \\
\hline
\end{tabular}

A diagram showing the list of filters with their the full width at the half-amplitude level, as well as some spectral lines that are correspond to the selected narrow-band filters, is shown in Fig. 4. 


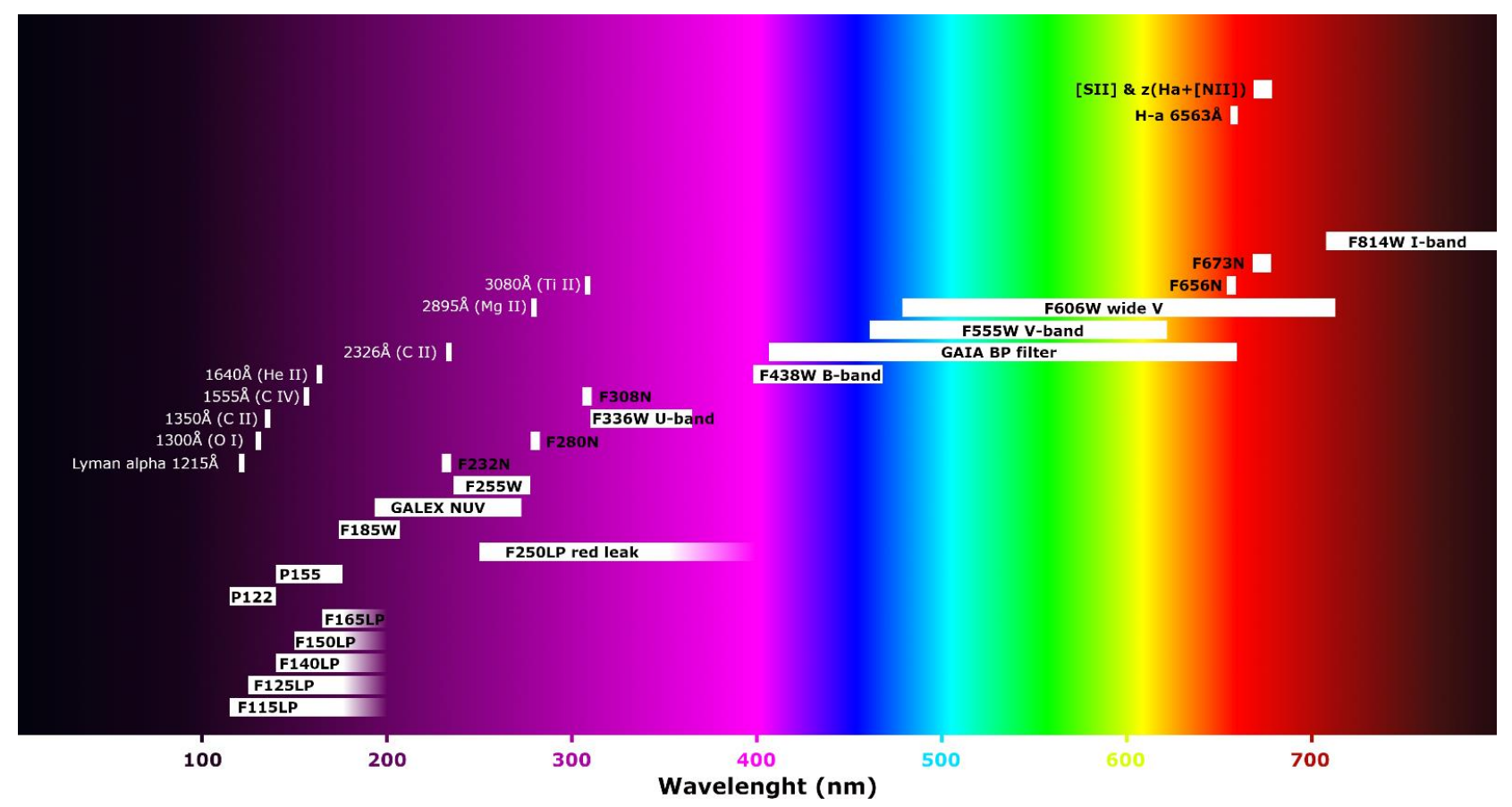

Figure 4. A diagram that shows the list of filters and the full width at the half-amplitude level, as well as some spectral lines that correspond to the selected narrow-band filters

\section{SUMMARY}

The Spektr-UF (WSO-UV) project is an efficient multipurpose orbital observatory for high and low resolution spectroscopy, high sensitivity imaging and slitless spectroscopy in the ultraviolet. The imaging instrument FCU onboard WSO-UV will be the first UV camera to be flown to a geosynchronous orbit. In the Federal Space Program of Russia 2016-2025 the launch of the project is scheduled for 2025.

Up to date information on the WSO-UV mission can be found on the web site of Joint Centre of Ultraviolet Astronomy: http://jcuva.space.

\section{REFERENCES}

[1] Sachkov, M., Sachkov, M., Shustov, B., \& Gómez de Castro, A.I., "Instrumentation of the WSO-UV project. DOI 10.1117/12.2055513," Proc. SPIE 9144, 914402 (2014)

[2] Sachkov, M., Shustov, B., Gómez de Castro, A. I., "World Space Observatory Ultraviolet mission: status 2016. DOI 10.1117/12.2233085," Proc. SPIE 9905, id. 990504, 6 pp. (2016)

[3] Sachkov, M., Shustov, B., Gómez de Castro, A. I., "World Space Observatory ultraviolet mission: instrumentation and the core program. DOI 10.1117/12.2312923," Proc. SPIE 10699, id. 106993G, 5 pp. (2018)

[4] Shustov, B., Sachkov, M., Gómez de Castro, A.I., "Comets in UV," Astrophysics and Space Science 363, article id. 64, (2018)

[5] Fossati, L., et al., "Major prospects of exoplanet astronomy with the World Space Observatory-UltraViolet mission," Astrophysics and Space Science 354, 9-19 (2014). 
[6] Sachkov, M.E., "Spectral studies of comets in the ultraviolet range and prospects of the WSO-UV project in these studies," Solar System Research 50, 294 - 299 (2016)

[7] Boyarchuk, A.A., et al., "Scientific problems addressed by the Spektr-UV space project (world space Observatory-Ultraviolet)," Astronomy Reports 60, 1 - 42 (2016)

[8] Malkov, O., Sachkov, M., Shustov, B., et al., "Scientific program construction principles and time allocation scheme for the World Space Observatory - Ultraviolet mission," Astrophys. Space Sci. 335, 323 - 327 (2011)

[9] Sachkov, M., et al., "The new field camera unit imaging instrument onboard WSO-UV. DOI 10.1117/12.2312933," Proc. SPIE 10699, id. 1069935, 4 pp. (2018) 